\title{
A set on which the local Łojasiewicz exponent is attained
}

\author{
by JACEK ChA̧DZYŃSKI and TADEUsz KRASiński (Łódź)
}

\begin{abstract}
Let $U$ be a neighbourhood of $0 \in \mathbb{C}^{n}$. We show that for a holomorphic mapping $F=\left(f_{1}, \ldots, f_{m}\right): U \rightarrow \mathbb{C}^{m}, F(0)=0$, the Lojasiewicz exponent $\mathcal{L}_{0}(F)$ is attained on the set $\left\{z \in U: f_{1}(z) \cdot \ldots \cdot f_{m}(z)=0\right\}$.
\end{abstract}

1. Introduction. In $\left[\mathrm{CK}_{2}\right]$ the authors showed that for a polynomial mapping $F=\left(f_{1}, \ldots, f_{m}\right): \mathbb{C}^{n} \rightarrow \mathbb{C}^{m}, n \geq 2$, the Lojasiewicz exponent $\mathcal{L}_{\infty}(F)$ of $F$ at infinity is attained on the set $\left\{z \in \mathbb{C}^{n}: f_{1}(z) \ldots \cdot f_{m}(z)=0\right\}$. The purpose of this paper is to prove an analogous result for the Łojasiewicz exponent $\mathcal{L}_{0}(F)$, where $F: U \rightarrow \mathbb{C}^{m}$ is a holomorphic mapping, $F(0)=0$ and $U$ is a neighbourhood of $0 \in \mathbb{C}^{n}$ (Thm. 1). From this result we easily obtain a strict formula for $\mathcal{L}_{0}(F)$ in the case $n=2$ and $m \geq 2$ in terms of multiplicities of some mappings from $U$ into $\mathbb{C}^{2}$ defined by components of $F$ (Thm. 2). It is a generalization of Main Theorem from $\left[\mathrm{CK}_{1}\right]$. The proof of this theorem has been simplified by A. Płoski in $[\mathrm{P}]$. His proof has been an inspiration to write this paper.

Theorem 1 is an important tool for investigation of the Lojasiewicz exponent for analytic curves having an isolated intersection point at $0 \in \mathbb{C}^{m}$. Using it, we shall give, in the next paper $\left[\mathrm{CK}_{3}\right]$, an effective formula for the Lojasiewicz exponent for such curves in terms of their parametrizations.

2. The Lojasiewicz exponent. Let $U \subset \mathbb{C}^{n}, n \geq 2$, be a neighbourhood of the origin, $F: U \rightarrow \mathbb{C}^{m}$ a holomorphic mapping, and $S \subset U$ an analytic set in $U$. Assume that $0 \in \mathbb{C}^{n}$ is an accumulation point of $S$. Put $N(F \mid S):=\left\{\nu \in \mathbb{R}_{+}: \exists A>0, \exists B>0, \forall z \in S,|z|<B \Rightarrow A|z|^{\nu} \leq|F(z)|\right\}$. Here $|\cdot|$ means the polycylindric norm. If $S=U$ we write $N(F)$ instead of $N(F \mid U)$.

1991 Mathematics Subject Classification: Primary 32S05.

Key words and phrases: holomorphic mapping, Łojasiewicz exponent.

This research was partially supported by KBN Grant No. 2 P03A 05010. 
By the Lojasiewicz exponent of the mapping $F \mid S$ at 0 we mean

$$
\mathcal{L}_{0}(F \mid S):=\inf N(F \mid S) .
$$

Analogously $\mathcal{L}_{0}(F):=\inf N(F)$.

The following can be shown (cf. [LT], $\S \S 5,6)$ :

Proposition 1. If $F \mid S$ has an isolated zero at $0 \in \mathbb{C}^{n}$, then $\mathcal{L}_{0}(F \mid S) \in$ $N(F \mid S) \cap \mathbb{Q}$. Moreover, there exists an analytic complex curve $\varphi:\{t \in \mathbb{C}:$ $|t|<r\} \rightarrow S$ such that $\varphi(0)=0$ and

$$
|F \circ \varphi(t)| \sim|\varphi(t)|^{\mathcal{L}_{0}(F \mid S)} \quad \text { as } t \rightarrow 0 .
$$

From the above proposition we easily get

Corollary 1. $\mathcal{L}_{0}(F \mid S)<\infty$ if and only if $F \mid S$ has an isolated zero at $0 \in \mathbb{C}^{n}$.

3. The main result. Now, we shall give the main result of this paper.

TheOrem 1. Let $U \subset \mathbb{C}^{n}, n \geq 2$, be a neighbourhood of the origin, and $F=\left(f_{1}, \ldots, f_{m}\right): U \rightarrow \mathbb{C}^{m}$ a holomorphic mapping with $F(0)=0$. Define $S:=\left\{z \in U: f_{1}(z) \cdot \ldots \cdot f_{m}(z)=0\right\}$. Then

$$
\mathcal{L}_{0}(F)=\mathcal{L}_{0}(F \mid S) .
$$

The proof will be given in Section 4 .

Immediately from Theorem 1 we obtain an effective formula for the Eojasiewicz exponent in the case $n=2, m \geq 2$, generalizing an earlier result of the authors ([CK $]$, Main Theorem).

Let us begin with some notations. Let $U$ be a neighbourhood of $0 \in \mathbb{C}^{2}$. Then: $\mu(f, g)$ is the intersection multiplicity of a holomorphic mapping $(f, g): U \rightarrow \mathbb{C}^{2} ; \widehat{h}$ is the germ of a holomorphic function $h: U \rightarrow \mathbb{C}$ in the ring $\mathcal{O}^{2}$ of germs of holomorphic functions at $0 \in \mathbb{C}^{2}$; ord $h$ stands for the order of $h$ at $0 \in \mathbb{C}^{2}$.

THEOREM 2. Let $U \subset \mathbb{C}^{2}$ be a neighbourhood of the origin, and $F=$ $\left(f_{1}, \ldots, f_{m}\right): U \rightarrow \mathbb{C}^{m}$ a holomorphic mapping with $F(0)=0$. Put $f:=$ $f_{1} \cdot \ldots \cdot f_{m}$. If $\widehat{f} \neq 0$ and $\widehat{f}=\widehat{h}_{1} \cdot \ldots \cdot \widehat{h}_{r}$ is a factorization of $\widehat{f}$ into irreducible germs in $\mathcal{O}^{2}$, then

$$
\mathcal{L}_{0}(F)=\max _{i=1}^{r} \frac{1}{\operatorname{ord} h_{i}} \min _{j=1}^{m} \mu\left(h_{i}, f_{j}\right) .
$$

Proof. Since our considerations are local, we may assume that $h_{i}$ are holomorphic in $U$ and $f=h_{1} \cdot \ldots \cdot h_{r}$ in $U$. Let $S:=\{z \in U: f(z)=0\}$ and $\Gamma_{i}:=\left\{z \in U: h_{i}(z)=0\right\}$. Hence

$$
S=\Gamma_{1} \cup \ldots \cup \Gamma_{r} .
$$


Define $\lambda_{i}:=\left(1 / \operatorname{ord} h_{i}\right) \min _{j=1}^{m} \mu\left(h_{i}, f_{j}\right)$. If $\lambda_{i}=\infty$ for some $i$, then (3) holds. So, assume that $\lambda_{i} \neq \infty, i=1, \ldots, r$. Then for every $i$ we have

$$
|F(z)| \sim|z|^{\lambda_{i}} \quad \text { as }|z| \rightarrow 0, z \in \Gamma_{i}
$$

Hence and from $(4), \mathcal{L}_{0}(F \mid S)=\max _{i=1}^{r} \lambda_{i}$. This and Theorem 1 give (3), which ends the proof.

4. Proof of the main theorem. The proof is given in two steps. In the first one we give the proof under some additional assumptions, whereas in the second step we show that these assumptions do not restrict our considerations.

First we fix some notations. For $z=\left(z_{1}, \ldots, z_{n}\right) \in \mathbb{C}^{n}, n \geq 2$, and for every $i \in\{1, \ldots, n\}$ we put $z_{i}^{\prime}:=\left(z_{1}, \ldots, z_{i-1}, z_{i+1}, \ldots, z_{n}\right)$. Additionally, we define $f:=f_{1} \cdot \ldots \cdot f_{m}$.

STEP 1. We assume that

(i) $(F \mid S)^{-1}(0)=\{0\}$,

(ii) ord $f<\infty$,

(iii) for every $i \in\{1, \ldots, n\}, f$ is (ord $f$ )-regular with respect to $z_{i}$.

Obviously $N(F) \subset N(F \mid S)$. To show (2) it suffices to prove

$$
N(F \mid S) \subset N(F) \text {. }
$$

It follows from (i) and Corollary 1 that $N(F \mid S)$ is not empty. Take an arbitrary $\nu \in N(F \mid S)$. Then there exist $A, B>0$ such that

$$
|F(\zeta)| \geq A|\zeta|^{\nu} \quad \text { for } \zeta \in S,|\zeta|<B
$$

From the assumptions of the theorem we have ord $f>0$ and ord $f_{j}>0$, $j=1, \ldots, m$. Then from (ii), (iii) we easily get $0<$ ord $f_{j}<\infty$ and

(7) for every $i, j, f_{j}$ is (ord $f_{j}$ )-regular with respect to $z_{i}$.

The Weierstrass Preparation Theorem and (iii) imply that for every $i \in$ $\{1, \ldots, n\}$ there exists a Weierstrass polynomial with respect to $z_{i}$ of degree ord $f$, associated with $f$. Denote it by $p^{(i)}$. Analogously from (7) for every $i, j$ there exists a Weierstrass polynomial with respect to $z_{i}$ of degree ord $f_{j}$, associated with $f_{j}$. Denote it by $p_{j}^{(i)}$. Then there exist $C, D>0$ and a polycylinder $\left\{z \in \mathbb{C}^{n}:|z|<r\right\} \subset U$ such that

$$
C\left|p^{(i)}(z)\right| \leq|f(z)| \leq D\left|p^{(i)}(z)\right| \quad \text { for }|z|<r, i=1, \ldots, n,
$$

and

(9) $\quad C\left|p_{j}^{(i)}(z)\right| \leq\left|f_{j}(z)\right| \leq D\left|p_{j}^{(i)}(z)\right|$

$$
\text { for }|z|<r, i=1, \ldots, n, j=1, \ldots, m \text {. }
$$


Put $P^{(i)}:=\left(p_{1}^{(i)}, \ldots, p_{m}^{(i)}\right)$. Then from (9) we get

$$
C\left|P^{(i)}(z)\right| \leq|F(z)| \leq D\left|P^{(i)}(z)\right| \quad \text { for }|z|<r, i=1, \ldots, n .
$$

Clearly, for every $i \in\{1, \ldots, n\}$,

$$
p^{(i)}=p_{1}^{(i)} \cdot \ldots \cdot p_{m}^{(i)} .
$$

Put $B_{1}:=\min (B, r)$. Then from (8) we have for every $i \in\{1, \ldots, n\}$,

$$
S \cap\left\{z \in \mathbb{C}^{n}:|z|<B_{1}\right\}=\left\{z \in \mathbb{C}^{n}:|z|<B_{1}, p^{(i)}(z)=0\right\} .
$$

Hence and from the Theorem on Continuity of Roots, applied to $p^{(i)}$, we find that there exists $\varrho, 0<\varrho \leq B_{1}$, such that for every $i \in\{1, \ldots, n\}$ we have

$$
\text { (12) }\left\{z \in S:\left|z_{i}^{\prime}\right|<\varrho,|z|<B_{1}\right\}=\left\{z \in \mathbb{C}^{n}:\left|z_{i}^{\prime}\right|<\varrho, p^{(i)}(z)=0\right\} \text {. }
$$

Put $d:=\max _{j=1}^{m}$ ord $f_{j}, A_{2}:=2^{-d} A(C / D), B_{2}:=\min \left(\varrho, B_{1}\right)$. Take an arbitrary $\dot{z} \in \mathbb{C}^{n},|z|<\mid<B_{2}$. There exists $i \in\{1, \ldots, n\}$ such that $|z| z|=| \dot{z}_{i}^{\prime} \mid$.

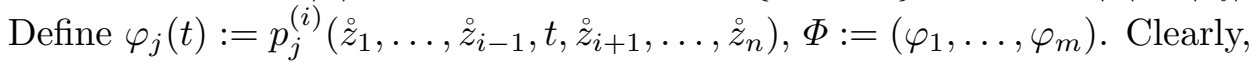
$\Phi: \mathbb{C} \rightarrow \mathbb{C}^{m}$ is a polynomial mapping, $\operatorname{deg} \Phi:=\max _{j=1}^{m} \operatorname{deg} \varphi_{j}=d$ and

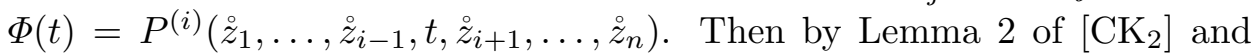
(11) we have

$$
\left|\Phi\left(\check{z}_{i}\right)\right| \geq 2^{-d} \min _{\tau \in T}|\Phi(\tau)|,
$$

where $T:=\left\{t \in \mathbb{C}: p^{(i)}\left(\check{z}_{1}, \ldots, \check{z}_{i-1}, t, \check{z}_{i+1}, \ldots, \check{z}_{n}\right)=0\right\}$. Hence and by (10) we get

$$
\begin{aligned}
|F(\check{z})| & \geq C\left|P^{(i)}(\stackrel{z}{z})\right|=C\left|\Phi\left(\check{z}_{i}\right)\right| \geq C 2^{-d}\left|\Phi\left(\tau_{0}\right)\right| \\
& =C 2^{-d}\left|P^{(i)}(\stackrel{\zeta}{\zeta})\right| \geq(C / D) 2^{-d}|F(\stackrel{\zeta}{\zeta})|
\end{aligned}
$$

for some $\stackrel{\wp}{\zeta}=\left(\check{z}_{1}, \ldots, \stackrel{\circ}{z}_{i-1}, \tau_{0}, \check{z}_{i+1}, \ldots, \check{z}_{n}\right)$ such that $p^{(i)}(\stackrel{\wp}{\zeta})=0$. Since $\left|\check{\zeta}_{i}^{\prime}\right|=$ $\left|z_{i}^{\prime}\right|=|\check{z}|<B_{2} \leq \varrho$, from (12) we have $\dot{\zeta} \in S$ and $|\dot{\zeta}|<B_{1}$. In consequence, from (6) and (13) we get

$$
|F(\check{z})| \geq(C / D) 2^{-d} A\left|\check{\zeta}^{\nu}\right|^{\nu} \geq A_{2}\left|\dot{\zeta}_{i}^{\prime}\right|^{\nu}=A_{2}|z|^{\nu}
$$

Since $z$ is arbitrary we obtain $\nu \in N(F)$. This ends the proof of the theorem under assumptions (i)-(iii).

SteP 2. Now, we shall prove the theorem in the remaining cases. If (i) does not hold, then by Corollary 1 we have $\mathcal{L}_{0}(F \mid S)=\mathcal{L}_{0}(F)=\infty$, that is, (2) is satisfied. If (ii) does not hold, then $S=U$ and (2) is obvious. So, it suffices to consider the case when (i), (ii) hold but (iii) does not. Since $N(F)$ and $N(F \mid S)$ are invariant with respect to linear automorphisms of $\mathbb{C}^{n}$, so are $\mathcal{L}_{0}(F)$ and $\mathcal{L}_{0}(F \mid S)$. Since the case considered can be reduced to Step 1 by a linear automorphism of $\mathbb{C}^{n},(2)$ also holds in this case.

This ends the proof of the theorem. 


\section{References}

[CK 1$]$ J. Chądzyński and T. Krasiński, The Eojasiewicz exponent of an analytic mapping of two complex variables at an isolated zero, in: Singularities, S. Lojasiewicz (ed.), Banach Center Publ. 20, PWN, Warszawa, 1988, 139-146.

$\left[\mathrm{CK}_{2}\right]-,-$, A set on which the Eojasiewicz exponent at infinity is attained, Ann. Polon. Math. 67 (1997), 191-197.

$\left[\mathrm{CK}_{3}\right]-$, - , On the Eojasiewicz exponent for analytic curves, in: Singularities Symposium-Łojasiewicz 70, B. Jakubczyk, W. Pawłucki and J. Stasica (eds.), Banach Center Publ., to appear.

[LT] M. Lejeune-Jalabert et B. Teissier, Clôture intégrale des idéaux et equisingularité, Centre de Mathématiques, École Polytechnique, 1974.

[P] A. Płoski, Newton polygons and the Eojasiewicz exponent of a holomorphic mapping of $\mathbb{C}^{2}$, Ann. Polon. Math. 51 (1990), 275-281.

Faculty of Mathematics

University of Łódź

Banacha 22

90-238 Łódź, Poland

E-mail: jachadzy@imul.uni.lodz.pl

krasinsk@krysia.uni.lodz.pl 